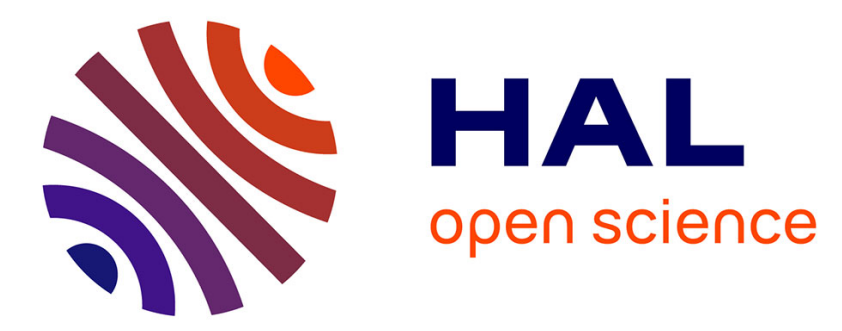

\title{
Real-time switching dynamics of ferroelectric tunnel junctions under single-shot voltage pulses
}

S. Boyn, A. Chanthbouala, S. Girod, C. Carretero, A. Barthelemy, M. Bibes, J. Grollier, S. Fusil, V. Garcia

\section{- To cite this version:}

S. Boyn, A. Chanthbouala, S. Girod, C. Carretero, A. Barthelemy, et al.. Real-time switching dynamics of ferroelectric tunnel junctions under single-shot voltage pulses. Applied Physics Letters, 2018, 113 (23), pp.232902. 10.1063/1.5054747 . hal-02288716

\section{HAL Id: hal-02288716 https://hal.science/hal-02288716}

Submitted on 12 Jul 2021

HAL is a multi-disciplinary open access archive for the deposit and dissemination of scientific research documents, whether they are published or not. The documents may come from teaching and research institutions in France or abroad, or from public or private research centers.
L'archive ouverte pluridisciplinaire HAL, est destinée au dépôt et à la diffusion de documents scientifiques de niveau recherche, publiés ou non, émanant des établissements d'enseignement et de recherche français ou étrangers, des laboratoires publics ou privés. 


\title{
Real-time switching dynamics of ferroelectric tunnel junctions under single-shot voltage pulses
}

\author{
S. Boyn ${ }^{1}$, A. Chanthbouala ${ }^{1}$, S. Girod ${ }^{1 *}$, C. Carrétéro ${ }^{1}$, A. Barthélémy ${ }^{1}$,

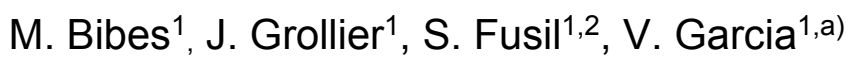 \\ ${ }^{1}$ Unité Mixte de Physique, CNRS, Thales, Univ. Paris-Sud, Université Paris-Saclay, 91767, \\ Palaiseau, France \\ ${ }^{2}$ Université d'Evry, Université Paris-Saclay, 91025 Evry, France.
}

In ferroelectric memory devices, information is stored within the polarization direction whose reversal usually occurs by the nucleation and propagation of domains. In ultrathin ferroelectrics, ultrafast dynamics may be achieved by nucleation-limited switching, avoiding the inherently speed-limited propagation of domain walls. Here we investigate polarization reversal dynamics in ultrathin ferroelectric films by transient current measurements. Thanks to the tunnel electroresistance, the start of polarization reversal induces sharp variations of the transmitted current under voltage pulses. These single-shot measurements show extremely fast switching with durations down to $3 \mathrm{~ns}$ that is only limited by the current device geometry. While the OFF-to-ON switching shows finite nucleation times that scale with the pulse amplitude, the ON-to-OFF

\footnotetext{
a) Author to whom correspondence should be addressed. Electronic mail: vincent.garcia@cnrs-thales.fr

* Now at Materials Research and Technology Department, Luxembourg Institute of Science and Technology (LIST), 41 rue du Brill, L-4422 Belvaux, Luxembourg
} 
switching speed cannot be detected under such rectangular pulses. Resorting to triangular pulse excitations allows to detect the dynamics of this switching direction. Both cases can be interpreted by nucleation switching models following Merz's law. 
The spontaneous polarization of ferroelectric materials can be reversed by the application of an electric field. As many potential applications of ferroelectrics are based on this property, ${ }^{1,2}$ understanding the dynamics of polarization switching is of crucial interest and has thus been the subject of many investigations, both in experiments as in theory. ${ }^{3-6}$ Piezoresponse force microscopy (PFM) imaging provides insights into static domain configurations ${ }^{7-9}$ and electrical measurements of the transient of displacement currents during switching under voltages pulses can reveal their temporal evolution ${ }^{1,10}$. These methods have helped to understand the polarization reversal process in ferroelectric bulk materials and films with thicknesses down to about $100 \mathrm{~nm}$. Here, we focus on the switching dynamics in ultrathin ferroelectric films.

In ferroelectric tunnel junctions (FTJs) an ultrathin ferroelectric layer is sandwiched between two metallic electrodes. When a voltage is applied across this capacitor-like structure, electrons can tunnel across the ferroelectric barrier. ${ }^{11}$ It has been shown that this tunnel current critically depends on the polarization orientation in the barrier, allowing for a resistive read-out of the ferroelectric polarization. ${ }^{12-14}$ Depending on the domain configuration, the device state can be varied from a high-resistance OFF state to a low-resistance ON state. FTJs can therefore be used as classical binary memories. ${ }^{15,16}$ Furthermore, mixed domain configurations lead to intermediate resistance states which opens the door to using FTJs as artificial synapses in neuromorphic hardware. ${ }^{17}$ For both applications, the polarization reversal dynamics under voltage pulses is of crucial importance.

The FTJs studied in this work are based on films of $\mathrm{BiFeO}_{3}(\mathrm{BFO}$, thickness $d=4.6 \mathrm{~nm}$ ) that are grown by pulsed laser deposition on $\mathrm{YAlO}_{3}$ substrates. ${ }^{18,19}$ An intermediate $\mathrm{Ca}_{0.96} \mathrm{Ce}_{0.04 \mathrm{MnO}}$ (CCMO) layer $(20 \mathrm{~nm})$ serves as bottom electrode and $\mathrm{Pt} / \mathrm{Co}$ is used as top electrode. Structuring of the electrodes produces fully patterned cells ${ }^{16}$ and allows for the application of short voltage 
pulses. The results shown here were obtained using circular top electrodes with diameters of 300 to $400 \mathrm{~nm}$.

Electrical dc measurements are conducted using a Yokogawa GS610 voltage source in series with a Keithley 6487 picoammeter and an Agilent $81150 \mathrm{~A}$ pulse generator is used to apply voltage pulses (Fig. 1c). A bias-tee (Mini-Circuits ZFBT-6GW+) combines the de and rf signals. The top electrode of the FTJ is connected to its output with an rf tee connector placed in between. As the FTJ resistance is much higher than the impedance of the $\mathrm{rf}$ components $(50 \Omega)$, the FTJ side of the rf tee connector is effectively equivalent to an open circuit which leads to $50 \Omega$ impedance matching at the tee. In this way, reflections should be very limited and we can record the voltage pulse as it occurs directly at the FTJ using an oscilloscope with $50 \Omega$ impedance (Agilent DSO9254A). The ground is made through the oscilloscope. The signal transmitted to the FTJ bottom electrode is split (Mini-Circuits ZFRSC-42) so that one part of the current is directly recorded by the oscilloscope ( $I_{\text {dir }}$ in Fig. 1 c). The second splitter output is fed into a current amplifier (Femto HCA-400M-5K-C) and this amplified signal (Iamp in Fig. 1c) is recorded by the oscilloscope in parallel. There are two different measurement modes: during pulsed single-shot measurements, the oscilloscope input recording the voltage pulse is set to an impedance of $50 \Omega$. For dc resistance measurements, the coupling of this input is changed to a purely capacitive mode in order to prevent the current from taking this path which presents a significant lower parallel resistance than the FTJ.

The raw transient current signal displays strong peaks at the start and the end of the applied rectangular voltage pulses. These can be attributed to the charging of a capacitor $(C)$, formed by the electrical contact lines, through the bottom electrode resistance $R_{\mathrm{s}}$. As a result, the temporal 
resolution of our experiment is limited to $R_{\mathrm{S}} C \approx 3 \mathrm{~ns}$ which is in good quantitative agreement with the sample geometry and the material properties. In order to reveal the intrinsic ferroelectric switching from the current signal, we subtract the data of a non-switching reference from all the measurements shown here, following the scheme of the classical positive-up-negative-down (PUND) method for polarization loops ${ }^{7,20}$. However, in FTJs, the strong tunneling current masks the rather weak signal of the displacement charges ${ }^{7,20}$ - that are strongly limited by the small size of capacitor - so that we cannot directly extract the reversed polarization.

Figure 1a shows a typical hysteresis of the dc resistance of these patterned FTJs measured at $V_{\mathrm{dc}}=-0.2 \mathrm{~V}$ after applying voltage pulses of $100 \mathrm{~ns}$ of varying amplitude. The OFF/ON ratio reaches values of 2,000. Figure $1 \mathrm{~b}$ shows the voltage at the FTJ during a 1-microsecond-long square voltage pulse with an amplitude of $V_{\mathrm{p}}=-2.15 \mathrm{~V}$. We notice a small amplitude decrease of about $0.1 \mathrm{~V}$ over the full pulse length which we attribute to the capacitance in the bias-tee. The discharging of this capacitance also accounts for the small voltage offset $(0.1 \mathrm{~V})$ at the end of the pulse. Before the pulse, the FTJ has been initialized to the OFF state with a dc resistance of $R_{\text {before }} \approx 10^{8} \Omega$ at $V_{\mathrm{dc}}=-0.2 \mathrm{~V}$. The transmitted current (Fig. 1b) is very low at the beginning of the pulse $\left(10 \mu \mathrm{A}\right.$, i.e. $\left.\approx 2 \times 10^{5} \Omega\right)$, indicating the non-linear variation of the current with voltage in the OFF state. After a delay time $t_{\text {delay }}=167 \mathrm{~ns}$, its amplitude abruptly increases. Following this sharp step, the amplitude slowly decreases. We attribute this close-to-exponential decay to the capacitance of the external circuit as mentioned above.

The dc resistance measured after the application of the voltage pulse ( $R_{\text {after }} \approx 10^{6} \Omega$ ) shows that it has led to a partial reversal of the polarization in the FTJ. The onset of this reversal can be assigned to the abrupt current increase during the pulse. Whereas the temporal resolution of the present setup does not allow us to resolve the inner details of this transition, we can extract the delay time 
$t_{\text {delay }}$ between the start of the voltage pulse and the onset of the current increase. As a result of the parallel transport mechanism of domains with low and high resistance, the reversal of a tiny fraction of the ferroelectric barrier already leads to a large resistance drop when the initial FTJ resistance is high (close to the OFF state). ${ }^{17,18}$ Thanks to this extreme sensitivity to the onset of the polarization reversal, we can identify the abrupt increase in current amplitude at $t_{\text {delay }}$ as the very beginning of the reversal, i.e. the appearance of a first reversed nucleus. ${ }^{21}$

When increasing the amplitude $V_{\mathrm{p}}$ of the voltage pulse by less than a volt (Fig. 2a), the delay time decreases by two orders of magnitude. Besides, we observe that the current amplitude after the switching event is not linked to the final resistance state. It exhibits a linear dependence on the applied voltage (Fig. 2b), indicating that the current is not limited by the tunneling barrier, which most likely exhibits Fowler-Nordheim tunneling under these high negative electric fields ${ }^{22-23}$. This is in line with previous transport characterizations that indicated a trapezoidal shape of the tunnel barrier, high at the $\mathrm{Co} / \mathrm{BiFeO}_{3}$ interface and low at the $\mathrm{BiFeO}_{3} / \mathrm{CCMO}$ interface ${ }^{24}$. Indeed, the series resistance of the patterned CCMO electrode (about $10^{4} \Omega$ ) seems to prevail ${ }^{25-27}$ and thus precludes any observation related to polarization changes in the barrier from the transient current signal.

Figure $2 \mathrm{c}$ shows the delay times for different voltage pulse amplitudes extracted from 20 repetitions each. Before each pulse, the dc resistance is initialized to $R_{\text {before }} \approx 10^{8} \Omega$ and the final resistances after the voltage pulses are around $R_{\text {after }} \approx 10^{5}$ to $10^{6} \Omega$. As a general trend, the delay decreases as the amplitude increases and its distribution becomes narrower. In the inset of Fig. 2c, the delay times are plotted on a logarithmic scale against the inverse of the electric pulse field $E_{\mathrm{p}}$, calculated as $E_{\mathrm{p}}=V_{\mathrm{p}} / d$. This representation shows that these two variables follow Merz's law ${ }^{28}$ 
$\left(t_{s} \propto t_{0} e^{-E_{a} / E_{p}}\right)$, which describes the switching time in ferroelectrics and can be related to nucleation processes ${ }^{4,29}$. A fit yields an activation field of $E_{\mathrm{a}}=(-1.80 \pm 0.11) \mathrm{V} / \mathrm{nm}$ and a time constant of $t_{0}=(1.1 \pm 0.5) 10^{-11} \mathrm{~s}$. Although the activation field is considerably larger than typical values of thicker ferroelectrics, comparably high values have been obtained on other ultrathin films and related to their highly strained nature..$^{17,29,30}$

We now focus on the other branch of the ferroelectric loop, i.e. the ON-to-OFF switching. When we initialize the FTJ to the ON state $\left(R_{\text {before }} \approx 10^{5} \Omega\right)$ and then apply rectangular voltage pulses of positive polarity (Fig. 3a), we cannot identify any delay time. The transmitted current is very low from the start of the pulse, indicating that the transition occurs at the very beginning (within the time resolution of $3 \mathrm{~ns}$ ). This very fast, abrupt switching from the $\mathrm{ON}$ to the OFF state can be explained considering the role of the series resistance $R_{\mathrm{S}}$ of the CCMO bottom electrode. At the start of the pulse, the applied voltage mainly drops at the series resistance and the voltage across the junction rises only slowly. However, as soon as the polarization reversal starts, the junction resistance increases. This in return then leads to a higher voltage drop across the junction which speeds up the switching process. As a result of this self-accelerating process, the switching from the ON to the OFF state is very abrupt.

As an alternative, we therefore resorted to triangular pulse shapes with long rise times that are characterized by their respective sweep rate $s_{\mathrm{p}}$. Under these, the current initially increases with

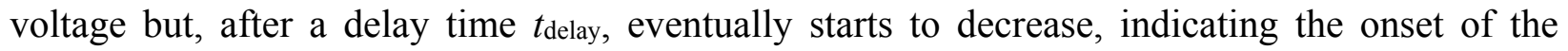
switching to higher resistance (Fig. 3b-d), analogous to the transition from the OFF state (Fig. 1b). When increasing the sweep rate from 11 to $32 \mathrm{~V} / \mu \mathrm{s}$ (Fig. $3 \mathrm{~b}$ to $\mathrm{d}$ ), the delay decreases as expected intuitively. Under pulses with higher sweep rates, a second current increase occurs towards the 
middle of the pulse (Fig. 3c-d). This reflects the strongly non-linear increase of the current with voltage $(>2.5 \mathrm{~V})$ in the tunneling regime and, consequently, does not indicate another switching event.

We conducted measurements of the delay times using sweep rates of up to $100 \mathrm{~V} / \mu \mathrm{s}$ (Fig. 4a). In order to test if these results are also compatible with Merz's law ${ }^{28}$ we use its equation in a Dirac distribution form and calculate its mean value $t_{\mathrm{s}}$ using a time-dependent voltage $V_{\mathrm{p}}(t)=s_{\mathrm{p}} t$ :

$$
\begin{aligned}
t_{\mathrm{s}}\left(s_{\mathrm{p}}\right) & =\int t \delta\left(t-t_{0} \exp \left\{\frac{E_{\mathrm{a}} d}{V_{\mathrm{p}}(t)}\right\}\right) \mathrm{d} t \\
& =\int t \delta\left(t-t_{0} \exp \left\{\frac{E_{\mathrm{a}} d}{s_{\mathrm{p}} t}\right\}\right) \mathrm{d} t \\
& =\int t \delta\left(t-\frac{E_{\mathrm{a}} d}{s_{\mathrm{p}}} / \mathrm{W}\left\{\frac{E_{\mathrm{a}} d}{s_{\mathrm{p}} t_{0}}\right\}\right) \mathrm{d} t \\
& =\frac{E_{\mathrm{a}} d}{s_{\mathrm{p}}} / \mathrm{W}\left\{\frac{E_{\mathrm{a}} d}{s_{\mathrm{p}} t_{0}}\right\} .
\end{aligned}
$$

Here, we employ the Lambert (W) function ${ }^{31}$ to express the root of the Dirac function. Using equation (1), we can reproduce the experimental data as a function of the sweep rate $s_{\mathrm{p}}$ with a very good agreement (black line in Fig. 4a) by fixing $t_{0}$ to the value extracted from the OFF-to-ON switching (inset of Fig. 2c). Both the nucleation times obtained by rectangular pulses and the data using different sweep rates can be described by Merz's law. This yields an activation field of $E_{\mathrm{a}}=1.83 \pm 0.02 \mathrm{~V} / \mathrm{nm}$ for the ON-to-OFF switching, very similar to the $E_{\mathrm{a}}=-1.80 \pm 0.11 \mathrm{~V} / \mathrm{nm}$ corresponding to the OFF-to-ON switching. However, the data can also be fitted with fixed $t_{0}$ values ranging from $10^{-15}$ to $10^{-10} \mathrm{~s}$ which results in activation fields between 3.9 and $1.3 \mathrm{~V} / \mathrm{nm}$ (yellow band in Fig. 4a). Due to this uncertainty, the quantitative agreement between the two reversal directions has to be considered with caution. We previously conducted stroboscopic PFM 
experiments and domain dynamics under cumulative pulses in similar FTJs which indicate a nucleation-limited switching behavior in which the nucleation times follow Merz's law. ${ }^{32}$ Such domain dynamics under cumulative pulses show average activation fields of $2.4 \pm 0.4 \mathrm{~V} / \mathrm{nm}$ for the ON-to-OFF switching. Thus, real-time transmission experiments and pseudo-static dynamics under cumulative pulses yield activation fields of comparable amplitudes.

The use of pulses with long rise times further allows us to determine the critical voltage $V_{\mathrm{c}}$ at which the polarization reversal starts. Figure $4 \mathrm{~b}$ shows this voltage as a function of the sweep rate. While the absolute variation of the critical voltage is rather low, it slightly increases with the sweep rate. This trend matches the findings obtained by classical hysteresis measurements which show that the critical electrical field (here: critical voltage) increases as a function of the driving frequency (analogous to the sweep rate here). ${ }^{33}$ The data furthermore agree with the critical-field dependency on the driving frequency calculated for a nucleation-limited process. ${ }^{33,34}$ This supports the assumption that the delay time corresponds to a first nucleation event in the polarization reversal.

In summary, we here presented the first investigation of polarization reversal dynamics in ultrathin ferroelectric films by transient current measurements. Thanks to the tunnel electroresistance effect, we could directly relate the electrical signal to polarization changes. The single-shot measurements show extremely fast switching with speeds up to 3 ns that is only limited by the current device fabrication design. Furthermore, we could identify the nucleation of a first reversed domain as the limiting process in the reversal dynamics. ${ }^{35,36}$ The nucleation time follows the classical Merz's law as a function of the applied pulse voltage. The observed differences concerning the reversal direction could be related to the built-in electric field ${ }^{24,37}$ or the influence of the bottom-electrode resistance. ${ }^{27}$ The approach reported here could be extended to other ferroelectric tunnel junctions and offers an insightful template to assess ultrafast switching dynamics in ferroelectrics. 
This publication has received funding from the European Union's Horizon 2020 research innovation program under grant agreement 732642 (ULPEC project). This work is supported by a public grant overseen by the French National Research Agency (ANR) as part of the 'Investissements d'Avenir' program (Labex NanoSaclay, reference: ANR-10-LABX-0035). We acknowledge financial support from the French Agence Nationale de la Recherche (ANR) projects FERROMON, MIRA and PIAF. We furthermore thank J. Sampaio for his help in designing the transmission measurement setup as well as E. Jacquet and C. Deranlot for technical assistance with the sample fabrication. 


\section{Figure captions}

Fig. 1. (a) Typical hysteresis of the dc resistance measured following 100-nanosecond voltage pulses of varying amplitude. (b) Evolution of the voltage ( $\left.V_{\text {pulse }}\right)$ and the transmitted current $\left(I_{\text {trans }}\right)$ signals during the application of a rectangular voltage pulse for the OFF-to-ON switching. The abrupt increase in current after a delay $t_{\text {delay }}$ can be associated with the nucleation of a first reversed polarization domain. (c) Circuit scheme used for single-shot transmission measurements.

Fig. 2. OFF-to-ON switching. (a) Time evolution of the transmitted current signals under voltage pulses of different amplitudes. From each, a delay time can be extracted. (b) Dependence of the maximum transmitted current after switching on the amplitude of the pulse. The linear relationship suggests that the series resistance $\left(R_{\mathrm{S}}\right)$ is dominating. (c) Dependence of the delay time on the voltage pulse amplitude. Open symbols show individual measurements and closed symbols indicate the mean value for each amplitude. Error bars correspond to the statistical standard deviation. In inset, mean delay times are shown as a function of the inverse applied electrical field. The black line is a fit according to Merz's law. Open symbols correspond to data that were excluded from the fitting process.

Fig. 3. ON-to-OFF switching. (a) Evolution of the voltage and the transmitted current signals during the application of a rectangular voltage pulse. (b-d) Evolution of the voltage and the transmitted currents signals during the application of triangular voltage pulses with different sweep 
rates $s_{\mathrm{p}}$. Due to the low initial resistance, the current increases at the beginning of the voltage ramp. When the polarization starts to reverse after a delay $t_{\text {delay }}$ (marked by a dotted vertical line), the current decreases. The corresponding critical voltage is indicated by a dashed horizontal line.

Fig. 4. ON-to-OFF switching. (a) Dependence of the delay time on the sweep rate of the triangular voltage pulses. Open symbols show individual measurements and closed symbols indicate the mean value for each sweep rate. The black line is a fit based on Merz's law using equation (1) with a fixed prefactor value of $t_{0}=1.1 \times 10^{-11} \mathrm{~s}$. The yellow band corresponds to fits for which $t_{0}$ was varied from $10^{-15}$ to $10^{-10} \mathrm{~s}$. (b) Dependence of the critical voltage on the sweep rate. Error bars correspond to the statistical standard deviation. 


\section{References}

${ }^{1}$ J.F. Scott and C.A.P.D. Araujo, Science 246, 1400 (1989).

2 J.F. Scott, Science 315, 954 (2007).

${ }^{3}$ M. Dawber, K.M. Rabe, and J.F. Scott, Rev. Mod. Phys. 77, 1083 (2005).

${ }^{4}$ Y.-H. Shin, I. Grinberg, I.-W. Chen, and A.M. Rappe, Nature 449, 881 (2007).

${ }^{5}$ A. Gruverman, J. Mater. Sci. 44, 5182 (2009).

${ }^{6}$ S.M. Yang, J.-G. Yoon, and T.W. Noh, Curr. Appl. Phys. 11, 1111 (2011).

${ }^{7}$ A. Gruverman, D. Wu, and J.F. Scott, Phys. Rev. Lett. 100, 97601 (2008).

${ }^{8}$ S.V. Kalinin, A.N. Morozovska, L.Q. Chen, and B.J. Rodriguez, Rep. Prog. Phys. 73, 56502 (2010).

${ }^{9}$ N. Balke, M. Gajek, A.K. Tagantsev, L.W. Martin, Y.-H. Chu, R. Ramesh, and S.V. Kalinin, Adv. Funct. Mater. 20, 3466 (2010).

${ }^{10}$ J. Li, B. Nagaraj, H. Liang, W. Cao, C.H. Lee, and R. Ramesh, Appl. Phys. Lett. 84, 1174 (2004).

${ }^{11}$ E. Y. Tsymbal and H. Kohlstedt, Science 313, 181 (2006).

${ }^{12}$ M.Y. Zhuravlev, R.F. Sabirianov, S.S. Jaswal, and E.Y. Tsymbal, Phys. Rev. Lett. 94, 246802 (2005).

${ }^{13}$ J. Velev, C.-G. Duan, K. Belashchenko, S. Jaswal, and E. Tsymbal, Phys. Rev. Lett. 98, (2007).

${ }^{14}$ V. Garcia, S. Fusil, K. Bouzehouane, S. Enouz-Vedrenne, N.D. Mathur, A. Barthelemy, and M. Bibes, Nature 460, 81 (2009).

15 A. Chanthbouala, A. Crassous, V. Garcia, K. Bouzehouane, S. Fusil, X. Moya, J. Allibe, B. Dlubak, J. Grollier, S. Xavier, C. Deranlot, A. Moshar, R. Proksch, N.D. Mathur, M. Bibes, and A. Barthélémy, Nat. Nanotechnol. 7, 101 (2012).

${ }^{16}$ S. Boyn, S. Girod, V. Garcia, S. Fusil, S. Xavier, C. Deranlot, H. Yamada, C. Carrétéro, E. Jacquet, M. Bibes, A. Barthélémy, and J. Grollier, Appl. Phys. Lett. 104, 52909 (2014). 
${ }^{17}$ A. Chanthbouala, V. Garcia, R.O. Cherifi, K. Bouzehouane, S. Fusil, X. Moya, S. Xavier, H. Yamada, C. Deranlot, N.D. Mathur, M. Bibes, A. Barthélémy, and J. Grollier, Nat. Mater. 11, 860 (2012).

${ }^{18}$ H. Yamada, V. Garcia, S. Fusil, S. Boyn, M. Marinova, A. Gloter, S. Xavier, J. Grollier, E. Jacquet, C. Carrétéro, C. Deranlot, M. Bibes, and A. Barthélémy, ACS Nano 7, 5385 (2013).

${ }^{19}$ H. Yamada, M. Marinova, P. Altuntas, A. Crassous, L. Bégon-Lours, S. Fusil, E. Jacquet, V. Garcia, K. Bouzehouane, A. Gloter, J.E. Villegas, A. Barthélémy, and M. Bibes, Sci. Rep. 3, 2834 (2013).

${ }^{20}$ J.F. Scott, L. Kammerdiner, M. Parris, S. Traynor, V. Ottenbacher, A. Shawabkeh, and W.F. Oliver, J. Appl. Phys. 64, 787 (1988).

21 A.K. Tagantsev, L.E. Cross, and J. Fousek, Domains in Ferroic Crystals and Thin Films (Springer New York, New York, NY, 2010).

${ }^{22}$ R.H. Fowler and L. Nordheim, R. Soc. Proc. Ser. Math. Phys. Eng. Sci. 119, 173 (1928).

${ }^{23}$ D. Pantel and M. Alexe, Phys. Rev. B 82, 134105 (2010).

${ }^{24}$ S. Boyn, V. Garcia, S. Fusil, C. Carrétéro, K. Garcia, S. Xavier, S. Collin, C. Deranlot, M. Bibes, and A. Barthélémy, APL Mater. 3, 61101 (2015).

${ }^{25}$ E.J. Guo, A. Herklotz, R. Roth, M. Christl, S. Das, W. Widdra, and K. Dörr, Appl. Phys. Lett. 103, 22905 (2013).

${ }^{26}$ F.Y. Bruno, S. Boyn, S. Fusil, S. Girod, C. Carrétéro, M. Marinova, A. Gloter, S. Xavier, C. Deranlot, M. Bibes, A. Barthélémy, and V. Garcia, Adv. Electron. Mater. 2, 1500245 (2016).

${ }^{27}$ S. Boyn, A.M. Douglas, C. Blouzon, P. Turner, A. Barthélémy, M. Bibes, S. Fusil, J.M. Gregg, and V. Garcia, Appl. Phys. Lett. 109, 232902 (2016).

${ }^{28}$ W.J. Merz, Phys. Rev. 95, 690 (1954).

${ }^{29}$ Y. Kim, H. Han, W. Lee, S. Baik, D. Hesse, and M. Alexe, Nano Lett. 10, 1266 (2010).

${ }^{30}$ N.A. Pertsev, J.R. Contreras, V.G. Kukhar, B. Hermanns, H. Kohlstedt, and R. Waser, Appl. Phys. Lett. 83, 3356 (2003). 
${ }^{31}$ R.M. Corless, G.H. Gonnet, D.E.G. Hare, D.J. Jeffrey, and D.E. Knuth, Adv. Comput. Math. 5, 329 (1996).

${ }^{32}$ S. Boyn, J. Grollier, G. Lecerf, B. Xu, N. Locatelli, S. Fusil, S. Girod, C. Carrétéro, K. Garcia, S. Xavier, J. Tomas, L. Bellaiche, M. Bibes, A. Barthélémy, S. Saïghi, and V. Garcia, Nat. Commun. 8, 14736 (2017).

${ }^{33}$ D.J. Jung, M. Dawber, J.F. Scott, L.J. Sinnamon, and J.M. Gregg, Integr. Ferroelectr. 48, 59 (2002).

${ }^{34}$ X. Du and I.-W. Chen, MRS Online Proc. Libr. Arch. 493, 311 (1997).

35 A. Tagantsev, I. Stolichnov, N. Setter, J. Cross, and M. Tsukada, Phys. Rev. B 66, 214109 (2002).

${ }^{36}$ J.Y. Jo, D.J. Kim, Y.S. Kim, S.-B. Choe, T.K. Song, J.-G. Yoon, and T.W. Noh, Phys. Rev. Lett. 97, $247602(2006)$.

${ }^{37}$ G. Gerra, A.K. Tagantsev, and N. Setter, Phys. Rev. Lett. 98, 207601 (2007). 


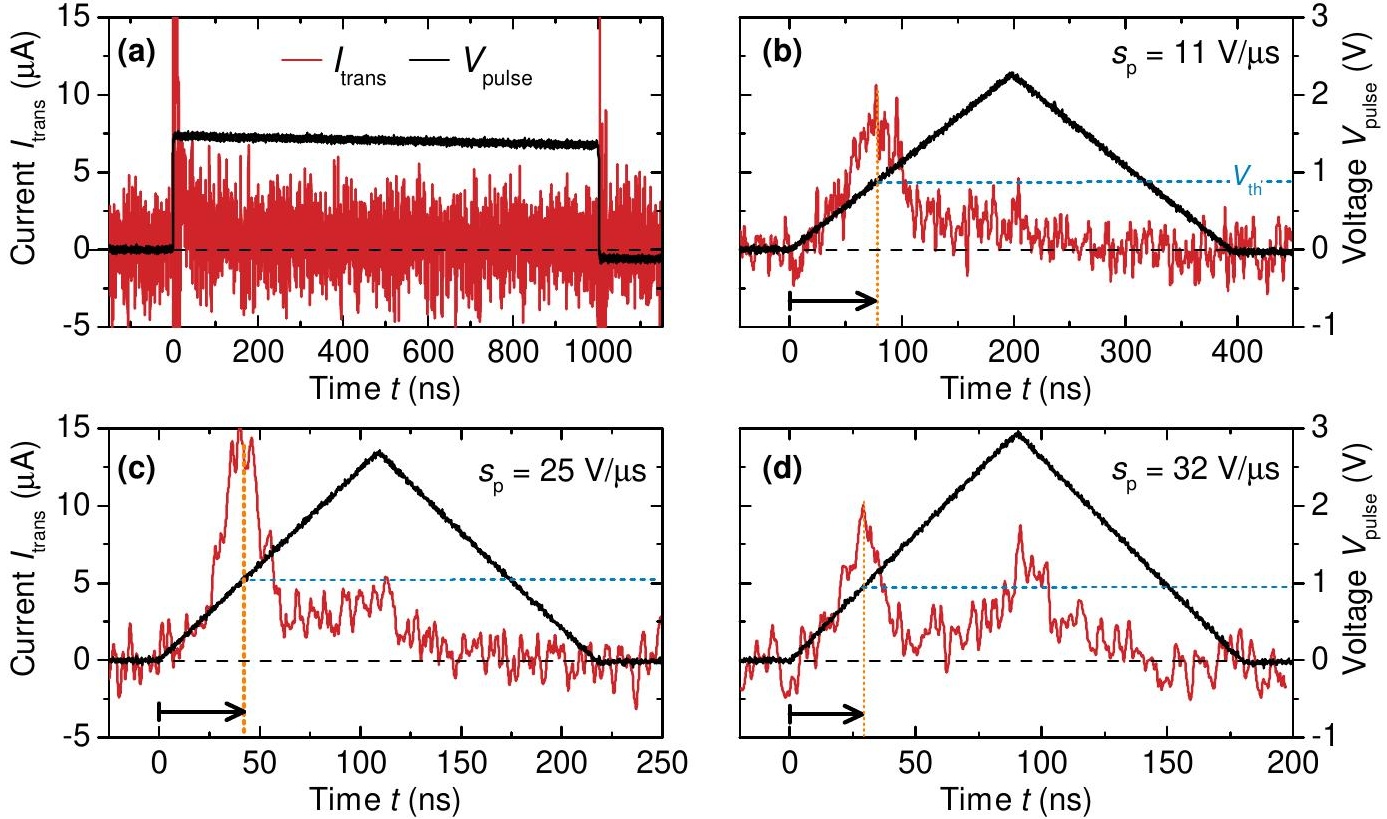


